\title{
Clinical, histological and genetic characterisation of patients with tubular aggregate myopathy caused by mutations in STIM1
}

\author{
Johann Böhm, ${ }^{1,2,3,4,5}$ Frédéric Chevessier, ${ }^{6}$ Catherine Koch, 1,2,3,4,5 \\ G Arielle Peche, $1,2,3,4,5$ Marina Mora, ${ }^{7,8}$ Lucia Morandi, ${ }^{7}$ Barbara Pasanisi, ${ }^{7}$ \\ Isabella Moroni, ${ }^{9}$ Giorgio Tasca, ${ }^{10}$ Fabiana Fattori, ${ }^{10}$ Enzo Ricci, ${ }^{11}$ \\ Isabelle Pénisson-Besnier, ${ }^{12}$ Aleksandra Nadaj-Pakleza, ${ }^{12}$ Michel Fardeau, ${ }^{13,14}$ \\ Pushpa Raj Joshi, ${ }^{15}$ Marcus Deschauer, ${ }^{15}$ Norma Beatriz Romero, ${ }^{13,14}$ \\ Bruno Eymard, ${ }^{13}$ Jocelyn Laporte $1,2,3,4,5$
}

For numbered affiliations see end of article.

\section{Correspondence to}

Dr Jocelyn Laporte, IGBMC, 1 Rue Laurent Fries, Illkirch 67404, France;

jocelyn@igbmc.fr, Isabelle Pénisson-Besnier died on July 11 th, 2013

Received 7 July 2014 Revised 23 September 2014 Accepted 25 September 2014 Published Online First 17 October 2014

\section{ABSTRACT}

Background Tubular aggregate myopathies (TAMs) are muscle disorders characterised by abnormal accumulations of densely packed single-walled or doublewalled membrane tubules in muscle fibres. Recently, STIM1, encoding a major calcium sensor of the endoplasmic reticulum, was identified as a TAM gene.

Methods The present study aims to define the clinical, histological and ultrastructural phenotype of tubular aggregate myopathy and to assess the STIM1 mutation spectrum.

Results We describe six new TAM families harbouring one known and four novel STIM1 mutations. All identified mutations are heterozygous missense mutations affecting highly conserved amino acids in the calcium-binding EFhand domains, demonstrating the presence of a mutation hot spot for TAM. We show that the mutations induce constitutive STIM1 clustering, strongly suggesting that calcium sensing and consequently calcium homoeostasis is impaired. Histological and ultrastructural analyses define a common picture with tubular aggregates labelled with Gomori trichrome and Nicotinamide adenine dinucleotide (NADH) tetrazolium reductase, substantiating their endoplasmic reticulum origin. The aggregates were observed in both fibre types and were often accompanied by nuclear internalisation and fibre size variability. The phenotypical spectrum ranged from childhood onset progressive muscle weakness and elevated creatine kinase levels to adultonset myalgia without muscle weakness and normal CK levels.

Conclusions The present study expands the phenotypical spectrum of STIM1-related tubular aggregate myopathy. STIM1 should therefore be considered for patients with tubular aggregate myopathies involving either muscle weakness or myalgia as the first and predominant clinical sign.

\section{INTRODUCTION}

Calcium $\left(\mathrm{Ca}^{2+}\right)$ is a physiological key factor and plays a pivotal role in a plethora of cellular functions. In skeletal muscle, $\mathrm{Ca}^{2+}$ triggers contraction and acts as a second messenger controlling growth and differentiation. Therefore, normal muscle function strongly depends on the tight regulation of $\mathrm{Ca}^{2+}$ storage and release. Store-operated calcium-entry (SOCE) efficiently couples sensing of stored $\mathrm{Ca}^{2+}$ with influx of extracellular $\mathrm{Ca}^{2+}$ and is the primary mechanism controlling intracellular $\mathrm{Ca}^{2+}$ homoeostasis in many cell types, including skeletal muscle. Store-operated calcium-entry is regulated by STIM1, a multidomain and single-pass transmembrane protein of the endoplasmic/sarcoplasmic reticulum. The luminal part of STIM1 consists of two EF hands and a sterile $\alpha$-motif (SAM), and the cytosolic C-terminus encompasses three coiled-coil domains, a serine/proline-rich segment and a lysine-rich segment. ${ }^{1-3}$ The stored $\mathrm{Ca}^{2}$ + is sensed by the EF hand domains and its binding maintains STIM1 in a closed conformation. ${ }^{3}$ At this resting state, STIM1 is evenly distributed at the endoplasmic reticulum/sarcoplasmic reticulum (ER/SR) membrane. Upon $\mathrm{Ca}^{2+}$ store depletion, the hydrophobic interaction between the EF and the SAM domains is weakened, allowing STIM1 to unfold and oligomerize. ${ }^{3}{ }^{4}$ The STIM1 oligomers translocate into punctae at contact sites between the ER/SR and the plasma membrane, where they activate the calcium release-activated calcium channels, resulting in extracellular $\mathrm{Ca}^{2+}$ entry. ${ }^{5-10}$

Mutations in STIM1 have been associated with three different disorders. Homozygous loss-of-function mutations were shown to cause immunodeficiency (MIM \#612783), ${ }^{11-13}$ whereas heterozygous gain-of-function mutations were found in patients with the multisystemic Stormorken syndrome (MIM \#185070) or with tubular aggregate myopathy (TAM, MIM \#160565). ${ }^{14-17}$ TAM is a progressive muscle disorder reported to be clinically heterogeneous, and either involving proximal muscle weakness, muscle cramps and pain, or myasthenic features ${ }^{18}$ rendering its clinical entity elusive. Biopsies from patients with TAM typically show regular arrays of membrane tubules. These aggregates appear as irregular red subsarcolemmal depositions on modified Gomori trichrome, and consist of densely packed single-walled or double-walled membrane tubules. Tubular aggregates are the only histopathological hallmark in tubular aggregate myopathy and were most often detected in type II muscle fibres. They also appear in muscle disorders as hypokalemic periodic paralysis ${ }^{19}$ or congenital limb-girdle myasthenia, ${ }^{20} 21$ and strikingly accumulate in normal muscle with age in mammals. ${ }^{22}$ 
In total, seven TAM families with STIM1 mutations have been reported to date. ${ }^{1617}$ Both studies focused on the genetic basis of the disease, while the clinical, histopathological and mutational spectra of STIM1-related TAM remain to be defined. Here we analyse and describe seven patients from six new and unrelated TAM families harbouring one known and four novel STIM1 mutations. All are heterozygous missense mutations affecting highly conserved amino acids in the EF hand domains and we demonstrate that they induce constitutive STIM1 clustering. Our study provides functional evidence for the pathogenesis of the identified STIM1 mutations and expands the clinical spectrum of STIM1-related tubular aggregate myopathy by describing a phenotypical TAM spectrum ranging from childhood-onset muscle weakness to adult-onset myalgia.

\section{MATERIALS AND METHODS}

\section{Patients}

Sample collection was performed with informed consent from the patients according to the declaration of Helsinki and experimentation was performed following institutional review board (IRB)-accepted protocols. Patients were from France (Families 1 and 6), Italy (Families 2, 3 and 4) and Germany (Family 5). Classification of tubular aggregate myopathy was based on histological and ultrastructural findings on muscle biopsies.

\section{Histology}

Transverse sections $(10 \mu \mathrm{m})$ of the muscle biopsies (deltoid for Families 1, 4 and 6, quadriceps for Families 2 and 3, and biceps brachii for Family 5) were stained with modified Gomori trichrome, NADH tetrazolium reductase (NADH-TR) and succinate dehydrogenase and assessed for fibre morphology, fibre type distribution and accumulation/infiltrations.

\section{Electron microscopy}

Muscle sections were fixed in 2.5\% paraformaldehyde, 2.5\% glutaraldehyde and $50 \mathrm{mM} \mathrm{CaCl}_{2}$ in $0.1 \mathrm{M}$ cacodylate buffer ( $\mathrm{pH}$ 7.4). Samples were postfixed with $2 \% \mathrm{OsO}_{4}, 0.8 \% \mathrm{~K}_{3} \mathrm{Fe}(\mathrm{CN})_{6}$ in $0.1 \mathrm{M}$ cacodylate buffer ( $\mathrm{pH} 7.4)$ for $2 \mathrm{~h}$ at $4^{\circ} \mathrm{C}$ and incubated with $5 \%$ uranyl acetate for $2 \mathrm{~h}$ at $4^{\circ} \mathrm{C}$. Muscles were dehydrated in a graded series of ethanol and embedded in epon resin. Thin sections were examined with an electron microscope (Philips CM120).

\section{Sequencing and segregation analysis}

Genomic DNA was prepared from peripheral blood by routine procedures and Sanger-sequenced for all coding exons of STIM1 and the adjacent splice-relevant regions. ${ }^{16}$ The mutations were numbered according to GenBank NM_001277961.1 and NP_001264890.1. Nucleotide position reflects cDNA numbering with +1 corresponding to the A of the ATG translation initiation codon. For the patient from Family 1, mutations in the Duchenne muscular dystrophy (DMD), COL6A1, COL6A and COL6A3 collagen genes were excluded. For the patient from Family 3, mutations in DMD and lamin A/C (LMNA) were excluded. Patient II.3 from Family 4 was screened for 98 muscle disease genes by highthroughput sequencing, and the patient from Family 5 was excluded for mutations in the phosphoglycerate mutase (PGAM) gene.

\section{Constructs}

The human STIM1-YFP (yellow fluorescent protein) construct was a kind gift from Nicolas Demaurex (University of Geneva, Switzerland). Point mutations (c.239A $>$ C, c.286C $>$ G, c.322T $>$ A, c.322T $>$ C and c.325C $>$ A) were introduced by sitedirected mutagenesis using the $P f u$ DNA polymerase (Stratagene, La Jolla, USA).

\section{Cell culture and imaging}

Murine C2C12 myoblasts were transfected with the STIM1-YFP constructs using Lipofectamine 2000 (Invitrogen, Carlsbad, USA) following the manufacturer's instructions, incubated with or without thapsigargin ( $1 \mu \mathrm{M}$; Sigma-Aldrich, St. Louis, USA) in Dulbecco's modified Eagle medium (DMEM) medium with $20 \%$ fetal calf serum (FCS), fixed and analysed by confocal microscopy (Leica SP2). For quantification, $>100$ cells/transfection were counted in three independent experiments and assessed for STIM1 clustering. Following antibodies were used for immunofluorescence: mouse Anti-p62 Lck ligand (BD Biosciences, San Jose, USA), mouse anti mono- and poly-ubiquitinylated conjugates (Enzo Life Sciences, Villeurbanne, France), rabbit anti-calnexin (Sigma-Aldrich, St. Louis, USA).

\section{RESULTS \\ Sequencing identifies four novel and one known STIM1 mutation}

Our TAM cohort encompassed patients from 38 unrelated families. All were suggestive of tubular aggregate myopathy, as tubular aggregates appearing in red on Gomori staining were the only histopathological hallmark. Sanger sequencing of all coding exons and adjacent splice-relevant regions of STIM1 revealed heterozygous mutations in six TAM families (figure 1A).

The patients from Families 1 and 2 harbour the same novel STIM1 c.239A >C (p.Asn80Thr) mutation in exon 2 (figure 1A). They are unrelated and originate from different countries. Detection of a known STIM1 polymorphism in exon 8 (rs2304891) in the heterozygous state only in the patient from Family 2 confirmed DNA individuality. The patient from Family 3 carries the novel STIM1 c. $286 \mathrm{C}>\mathrm{G}$ (p.Leu96Val) mutation in exon 2. The patients from Family 4 harbour the novel STIM1 c.322T >A (p.Phe108Ile) mutation in exon 3, affecting the same nucleotide as the novel mutation found in the patient from Family 5 (c.322T $>$ C; p.Phe108Leu). Genetic screening of the patient from Family 6 revealed the STIM1 c.325C >A (p.His109Asn) mutation in exon 3. This mutation has previously been described in another unrelated patient with TAM. ${ }^{16}$

Evidence of pathogenicity of the detected mutations comes from segregation analysis, as all affected, but none of the available unaffected family members carried the respective STIM1 mutations. For the sporadic cases in Families 1 and 3, DNA samples from both healthy parents were available and demonstrated de novo appearance of the mutations in the index patients. For the sporadic case in Family 2, DNA samples of all three living unaffected siblings were available. For Family 4, DNA samples were available for unaffected for the unaffected sibling and the unaffected father. For Family 5, DNA from the unaffected sister was available and for Family 6, only the index case was sequenced. However, the fact that the same mutation has been reported in an unrelated TAM family ${ }^{16}$ is a strong proof of pathogenicity. None of the mutations was listed in the single nucleotide polymorphism (SNP) databases as dbSNP, Exome Variant Server or the 1000 genomes project, or in our inhouse database comprising 416 exomes of individuals of similar ethnic origin as our patients.

In line with previous studies reporting STIM1 mutations in TAM, ${ }^{16}{ }^{17}$ all identified mutations were heterozygous missense mutations in exons 2 and 3. All published and new STIM1 mutations cluster in the $\mathrm{Ca}^{2+}$-binding EF hands and affect highly conserved amino acids (figure $1 \mathrm{~B}, \mathrm{C}$ ). On the resolved protein structure of the EF-SAM domain, ${ }^{3}$ mutations reside either in proximity to the $\mathrm{Ca}^{2+}$-binding site, or in the hydrophobic cleft implicated in the EF-SAM interaction to maintain the closed STIM1 conformation (figure 1D). 


\section{Genotype-phenotype correlations}

A

Family 1
c.239A $>$ C
p.Asn80Thr

Family 1
c.239A $>$ C

p.Asn80Thr

I

II
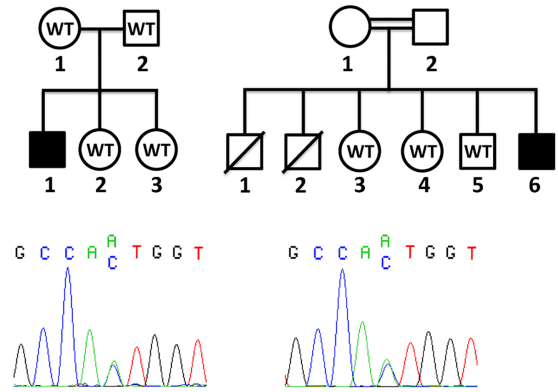

\section{Family 2}

c.239A $>C$

p.Asn80Thr

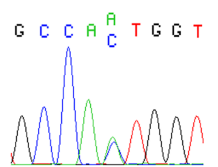

Family 3

c. $286 \mathrm{C}>\mathrm{G}$

p.Leu96Val
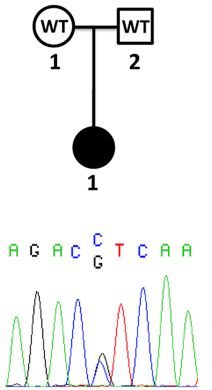

Family 4

c.322 $\mathrm{T}>\mathrm{A}$

p.Phe108Ile

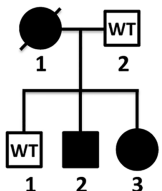

CACC ${ }_{A}^{T} T C C A$

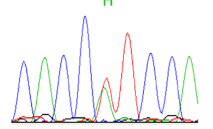

\section{Family 5 \\ c.322T $>$ C}

p.Phe108Leu

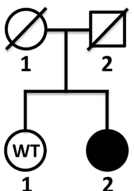

CAC C $C_{C}^{T} \mathrm{CCA}$

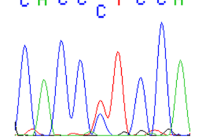

Family 6

c. $325 \mathrm{C}>\mathrm{A}$

p.His109Asn

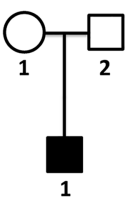

C T T C ${ }_{\text {A }}^{C}$ T G G

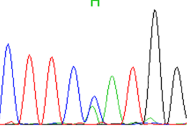

B

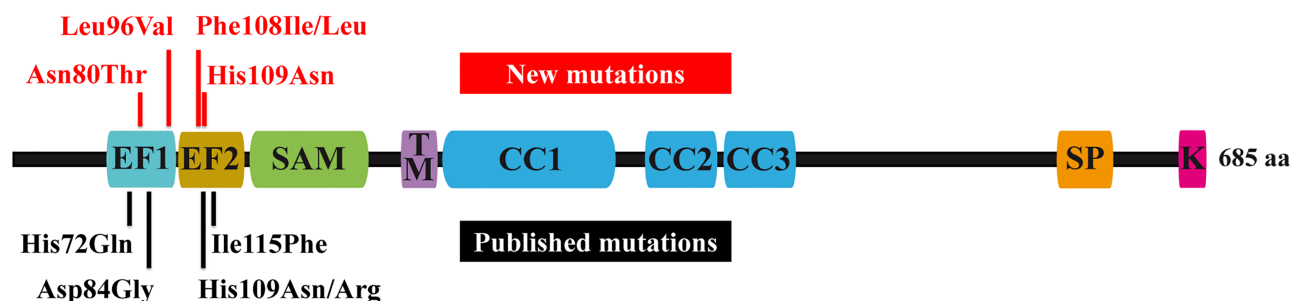

C

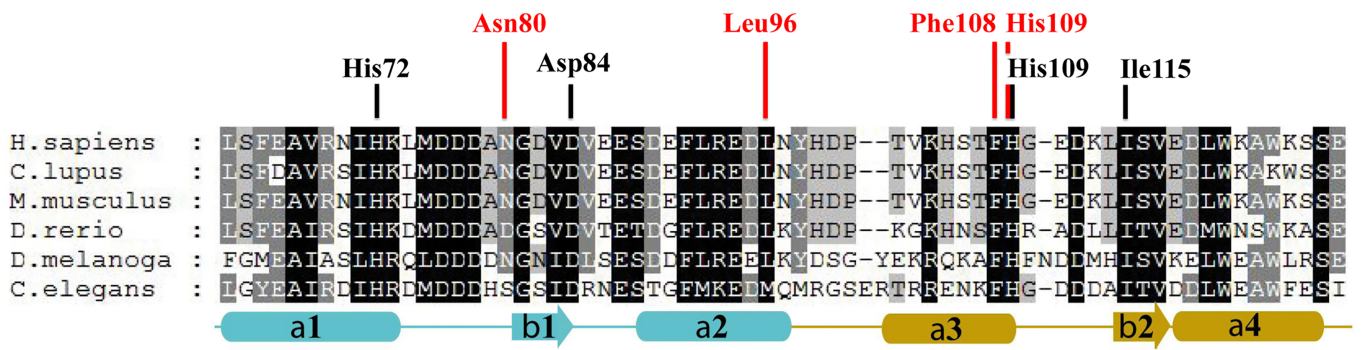

D
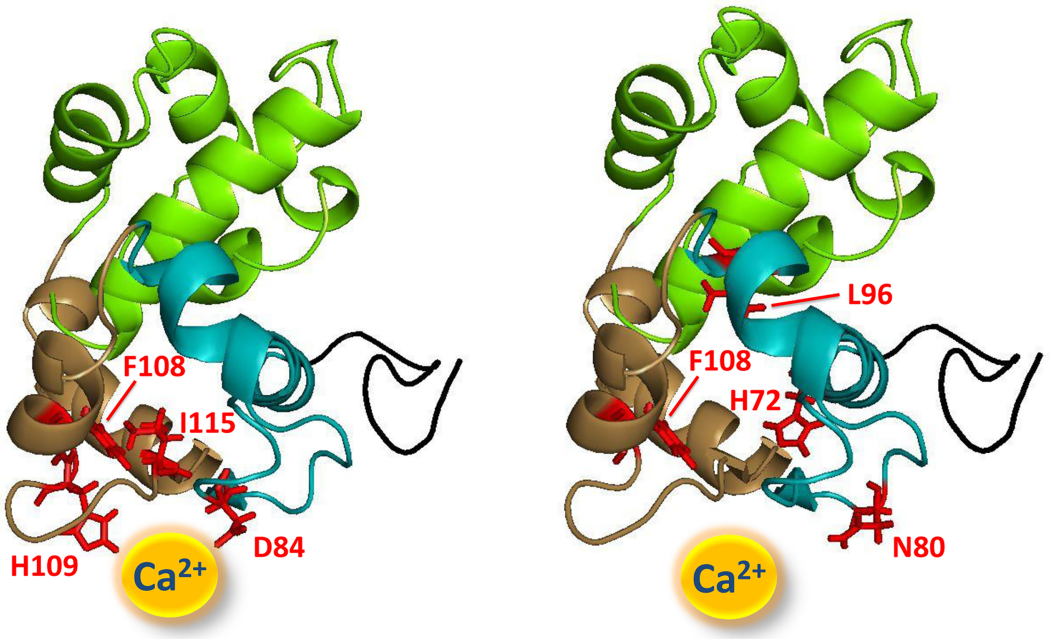

Figure 1 Identification of new STIM1 mutations in tubular aggregate myopathy. (A) Pedigrees of the six families with tubular aggregates myopathy and electropherograms showing the heterozygous STIM1 mutations. Healthy individuals without mutation in STIM1 are labelled as wild type (WT). DNAs from other family members were not available. (B) Schematic representation of the STIM1 protein domains. The previously reported STIM1 mutations are shown in black, the new STIM1 mutations in red. All mutations cluster in the intraluminal EF hands. TM, transmembrane domain; CC, coiled-coil; SP, serine/proline-rich domain; K, polylysines. (C) Protein sequence alignment of the STIM1 EF-hands of different species demonstrates high conservation of the mutated amino acids. (D) The resolved protein structure of the EF hands shows close proximity of the affected amino acids to the $\mathrm{Ca}^{2+}$ binding domain, especially for mutations involving muscle weakness as first clinical sign (left). The other mutations are depicted on the right picture. The EF1 appears in blue, the EF2 domain in brown and the sterile $\alpha$-motif (SAM) domain in green. 
Mutations induce STIM1 clustering independent of intraluminal $\mathrm{Ca}^{2+}$ level

Missense mutations in the EF hands were previously shown to induce constitutive STIM1 oligomerisation, probably as a result of decreased $\mathrm{Ca}^{2+}$ sensing. ${ }^{16}$ In order to assess the impact of the novel STIM1 mutations, we transfected C2C12 myoblasts with the different STIM1-YFP constructs. Addition of the SERCA1 inhibitor thapsigargin induced sarcoplasmic reticulum $\mathrm{Ca}^{2+}$ depletion and resulted in clustering of wild type STIM1 (figure 2). In line with the previously published STIM1 mutations, the myoblasts transfected with any of the new mutant constructs displayed statistically significant STIM1 clustering independently of thapsigargin, evidencing the pathogenicity of the novel STIM1 mutations described here. Of note, the Phe108Leu mutation involved less prominent (but still significant) STIM1 clustering compared with the other mutants. We next analysed the subcellular localisation of the constitutive STIM1 clusters and their potential association with autophagy. Monitoring the reticulum marker calnexin, and the autophagic markers p62 and ubiquitin did not reveal major differences between cells expressing wild type or mutant STIM1 (figure 3). The STIM1 clusters were found to reside within the sarcoplasmic reticulum and did not involve autophagy. These results strongly suggest that the constitutive STIM1 clusters preserve their functional activity.

\section{STIM1 mutations are associated with a large phenotypical spectrum}

The patients from Families 1 and 2 carry the same STIM1 mutation (p.Asn80Thr) and both had a similar course of disease (table 1). The patient from Family 1 presented with a childhood-onset postexercise myalgia and fatigability, associated with elevated creatine kinase (CK) levels. Mild proximal muscle weakness of the lower limbs appeared at age 35 years. The patient from Family 2 presented with adult-onset myalgia and elevated CK levels, and progressively developed muscle weakness, heel contractures, and reduced lateral and upward gaze. Owing to the age of both patients at the last medical examination (35 years vs 70 years), it is conceivable that the novel STIM1 p.Asn80Thr mutation first involves myalgia and high $\mathrm{CK}$, while additional TAM features as muscle weakness and eye movement defects appear at later stages of the disease. Similarly, the patient from Family 3 (p.Leu96Val) presented with childhood-onset myalgia and elevated CK as first symptoms and then developed muscle weakness predominantly affecting the lower limbs.

The STIM1 mutations found in Families 4 and 5 affect the same nucleotide (c.322T $>\mathrm{A}$ and c.322T $>\mathrm{C}$ ), and result in the integration of the chemically similar amino acids isoleucine and leucine (p.Phe108Ile and p.Phe108Leu). The clinical presentation is however different. The patients from Family 4 had childhood-onset limb-girdle muscle weakness. Myalgia appeared as secondary feature and was more pronounced in patient II.3. In contrast, the patient from Family 5 had adult-onset myalgia without muscle weakness and was the only patient from our cohort with normal CK levels.

The patient from Family 6, carrying the p.His109Asn mutation, presented with exercise intolerance, fatigability and high CK levels, but myalgia was not reported. His mother was referred to have an adult-onset muscle weakness of the lower limbs, but maternal DNA was not available for STIM1 sequencing. Noteworthy, the same p.His109Asn mutation has previously been described in an unrelated TAM family with muscle weakness as predominant clinical sign. ${ }^{16}$
In summary, the novel STIM1 p.Asn80Thr, p.Leu96Val and p. Phe108Leu mutations appear to be associated with myalgia as a first and major clinical sign. In contrast, the novel p.Phe108Ile and all previously reported STIM1 mutations (p.Asp84Gly, p. His109Asn, p.His109Arg and p.Ile115Phe) mainly involve proximal muscle weakness. ${ }^{16} 17$ Interestingly, on the resolved protein structure of the STIM1 EF-SAM domain, the mutations inducing muscle weakness cluster in the $\mathrm{Ca}^{2+}$-binding site (figure 1D). However, the novel p.Phe108Ile, involving myalgia without muscle weakness, also affects an amino acid in the $\mathrm{Ca}^{2}$ ${ }^{+}$-binding site. A genotype-phenotype correlation can therefore only partially be deduced from the three-dimensional model.

\section{Histopathological characteristics}

Muscle biopsies from all patients described in this study displayed tubular aggregates appearing in red on Gomori trichrome and in dark blue on NADH-TR staining (figure 4 and table 1). The aggregates were not detected with succinate dehydrogenase staining, indicating a reticulum and not a mitochondrial origin, as previously suggested. ${ }^{23}{ }^{24}$ Aggregates were observed in both fibre types in all patients, although they were predominantly detected in type II fibres in the patient from family 1, and in type I fibres in the patient from Family 3. Fibre size variability and internalised nuclei were seen in the patients from families $1,2,3$ and 4 and type II fibre atrophy was noted in patients from families 5 and 6 .

Ultrastructural analysis demonstrated that the tubular aggregates are of variable size and consist of single-walled or doublewalled membranes of different diameters (figure 5). We found vesicular membrane collection-type tubular aggregates in Families 1 and 6, Ic-type aggregates in Families 2, 3, 4 and 6, as well as Ib-type aggregates in Family 4. There was no obvious correlation between the tubular aggregate types and mutation, age of onset, or disease severity.

\section{DISCUSSION}

This study provides a clinical, histological, genetic and functional characterisation of six new families with tubular aggregate myopathy and mutations in STIM1, and their comparison with all previously published cases. We discovered four novel and one known STIM1 mutation. Three novel STIM1 mutations were associated with myalgia as a first and predominant clinical sign, and one involved a mixed muscle weakness/myalgia phenotype. At the molecular level, all STIM1 mutations found in our patients induced constitutive STIM1 clustering. This study provides functional evidence for the pathogenesis of the identified STIM1 mutations, and significantly expands the clinical spectrum of STIM1-related tubular aggregate myopathy.

\section{Clinical spectrum}

TAM has been reported with dominant and recessive inheritance and is therefore believed to be genetically heterogeneous. This is strengthened by the clinical heterogeneity of TAM, encompassing three major and distinct phenotypes. ${ }^{18}$ The first phenotype is characterised by a slowly progressive weakness predominantly affecting the proximal muscles, the second phenotype primarily involves myalgia with or without cramps, and the third phenotype associates a myopathic pattern with myasthenic features and is referred to as limb-girdle myasthenia.

With the exception of one family without apparent clinical phenotype (p.His72Gln), all previously reported patients with TAM with STIM1 mutations (p.Asp84Gly, p.His109Asn/Arg and p.Ile115Phe) presented with progressive muscle weakness and therefore belong to the first phenotypical TAM group. ${ }^{16} 17$ 

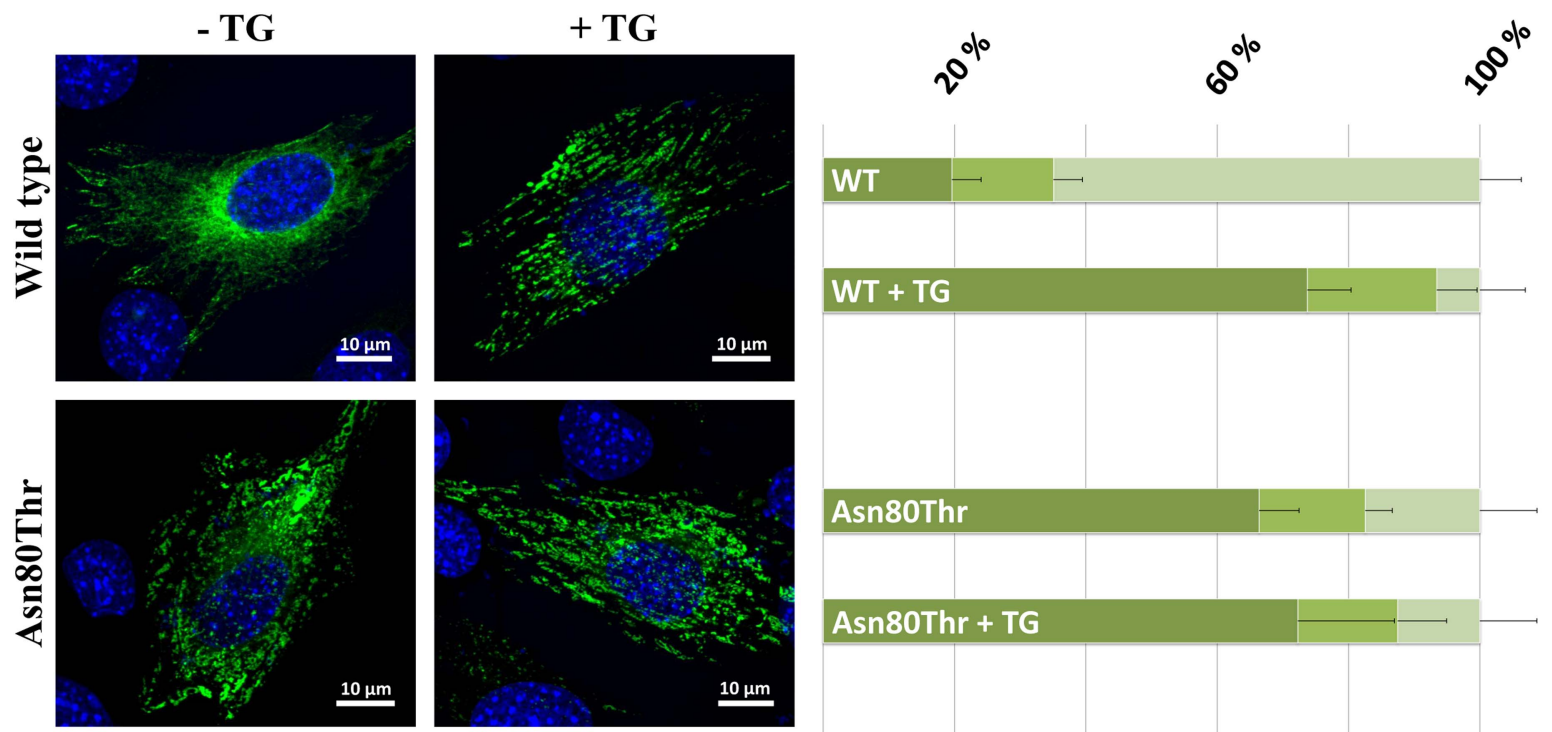

$\mathrm{WT}+\mathrm{TC}$
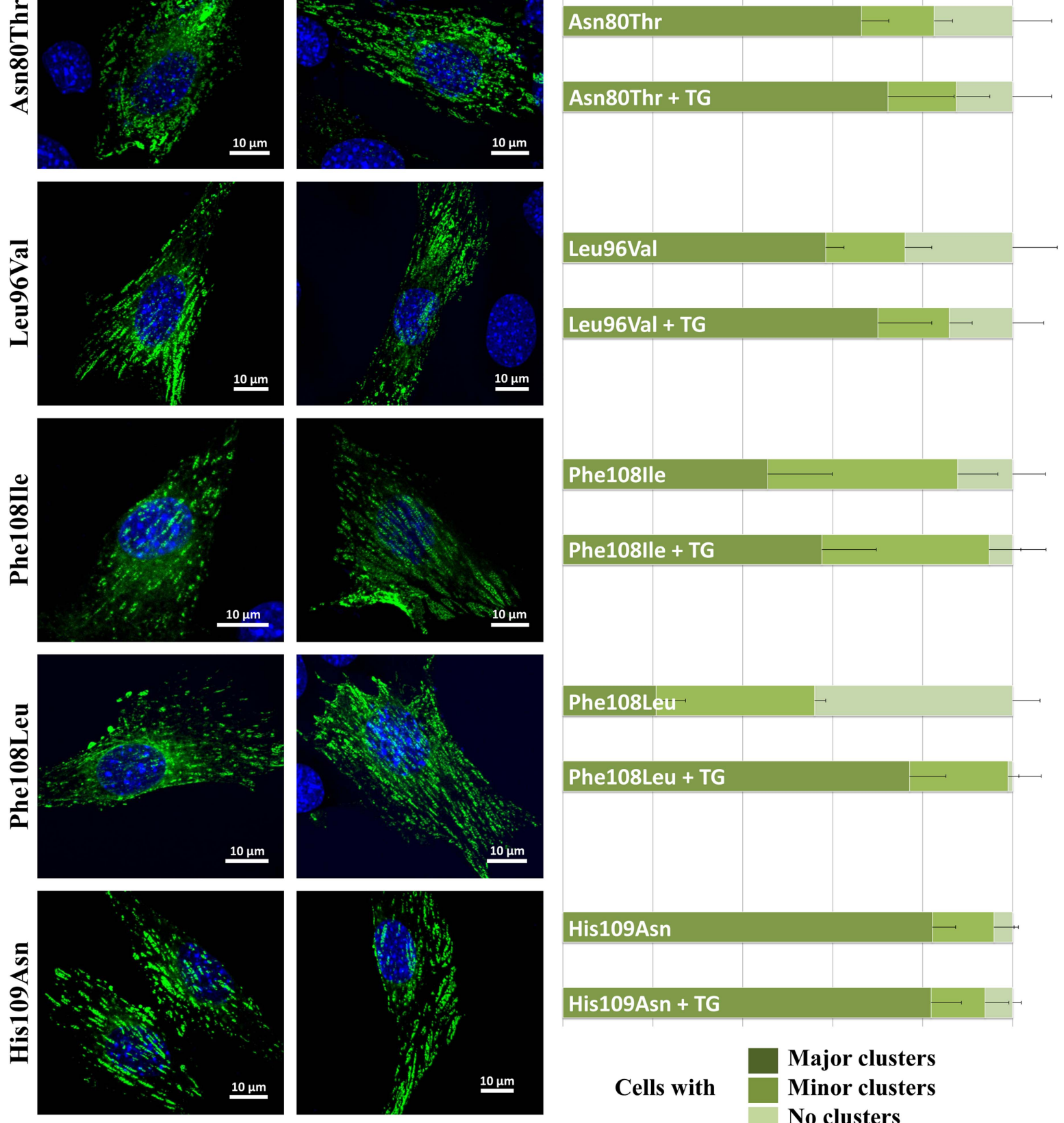

His109Asn + TG

Cells with

Major clusters
Minor clusters
No clusters

Figure 2 STIM1 clustering in muscle cells in dependence of thapsigargin (TG) and quantification. C2C12 myoblasts were transfected with the different STIM1-YFP constructs. Wild type STIM1 was evenly distributed in the ER and clustered upon store depletion induced by thapsigargin. All mutant constructs clustered independently of TG. Significant STIM1 clustering without TG was seen for all mutants and to a lesser extent for the construct harbouring the Phe108Leu mutation. Total number of counted cells ( $n>100$ ) was set to 100\%. Dark green bars correspond to the percentage of cells with major clusters, medium green bars represent the percentage of cells with minor clusters, and light green bars represent the percentage of cells without clusters. Error bars represent SD. 
Figure 3 The STIM1 clusters observed in myoblasts overexpressing mutant STIM1 constructs were negative for ubiquitin or the autophagic marker p62, and overall autophagic activity was similar in cells expressing wild type or mutant STIM1. Monitoring the reticulum using calnexin did not reveal significant differences in the SR structure in transfected or non-transfected cells. The other mutations N80T, L96V and $\mathrm{F} 108 \mathrm{I} / \mathrm{L}$ gave the same results (not shown).
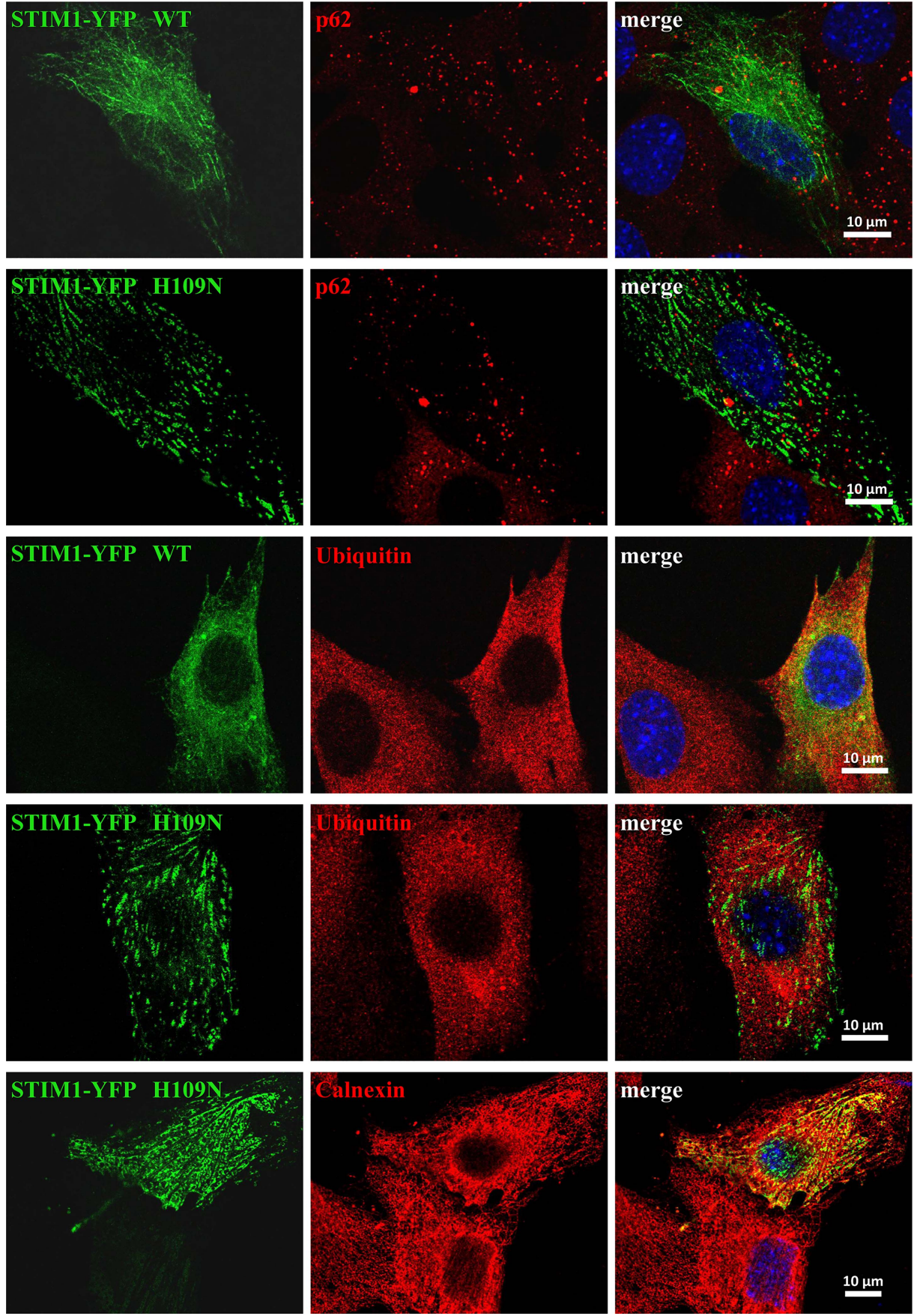

This study now describes seven new patients with TAM from six families harbouring known and novel STIM1 mutations. The phenotype of the patient with the known p.His109Asn mutation involved exercise intolerance and fatigability, and the patient's mother was reported with muscle weakness. Similarly, patients from Family 4 with p.Phe108Ile mutations first displayed muscle weakness before presenting a secondary myalgia. In contrast, the patients from the other four families harbour new STIM1 mutations (p.Asn80Thr, p.Leu96Val, Phe108Leu), and all presented with myalgia as the first symptom. Three of the patients developed muscle weakness and other secondary clinical features such as contractures, reduced tendon reflexes or eye movement disabilities at later disease stages. These data indicate that specific STIM1 mutations may cause distinct TAM phenotypes with different course of disease.
There is no domain-specific clustering of STIM1 mutations involving either muscle weakness or myalgia and these different phenotypes can even result from mutations in the same amino acid as Phe108. On the resolved protein structure, ${ }^{3}$ Asp84 belongs to the negatively charged calcium-binding site and mutation of this amino acid most probably interferes with $\mathrm{Ca}^{2+}$ sensing and thereby triggers STIM1 oligomerisation. Similarly, Phe108, His109 and Ile115 are on a loop in the $\mathrm{Ca}^{2+}$ binding region. In contrast, Leu96 resides within the hydrophopic cleft interacting with the SAM domain. Upon $\mathrm{Ca}^{2+}$ store depletion, the hydrophobic cleft becomes solvent-exposed, which destabilises the EF-SAM interaction. In this model, the Leu96 mutation is expected to impact on the intramolecular EF-SAM interaction irrespective of $\mathrm{Ca}^{2+}$ sensing, mediating constitutive activation of STIM1. The different intramolecular localisation of the STIM1 
Table 1 Molecular, phenotypical and histopathological data of patients with TAM with STIM1 mutations

\begin{tabular}{|c|c|c|c|c|c|c|c|}
\hline & Family 1 & Family 2 & Family 3 & Family 4 & & Family 5 & Family 6 \\
\hline Individual & II.1 & II.4 & II.1 & 11.2 & 11.3 & II.2 & II.1 \\
\hline Gender & M & M & $\mathrm{F}$ & M & $\mathrm{F}$ & $\mathrm{F}$ & M \\
\hline Mutation & $\begin{array}{l}\text { c.239A }>C \\
\text { p.Asn80Thr }\end{array}$ & $\begin{array}{l}\text { c.239A }>C \\
\text { p.Asn80Thr }\end{array}$ & $\begin{array}{l}\text { c. } 286 C>G \\
\text { p.Leu96Val }\end{array}$ & $\begin{array}{l}\text { c.322T }>A \\
\text { p.Phe108lle }\end{array}$ & $\begin{array}{l}\text { c.322T>A } \\
\text { p.Phe108lle }\end{array}$ & $\begin{array}{l}\text { c.322T>C } \\
\text { p.Phe108Leu }\end{array}$ & $\begin{array}{l}\text { c. } 325 C>A \\
\text { p. His109Asn }\end{array}$ \\
\hline Onset & Childhood & Adulthood & Childhood & Childhood & Childhood & Adulthood & Childhood \\
\hline $\begin{array}{l}\text { Age at last } \\
\text { examination }\end{array}$ & 34 & 70 & 13 & 51 & 46 & 52 & 35 \\
\hline Muscle weakness & Proximal, lower limbs & $\begin{array}{l}\text { Proximal, predominantly } \\
\text { lower limbs }\end{array}$ & Lower limbs & Predominantly proximal & Predominantly proximal & No & No \\
\hline Walking & Normal & $\begin{array}{l}\text { Foot drop, unable to walk } \\
\text { on heels }\end{array}$ & Normal & $\begin{array}{l}\text { With support, unable to walk on } \\
\text { heels }\end{array}$ & $\begin{array}{l}\text { With support, unable to walk on } \\
\text { heels }\end{array}$ & Normal & Normal \\
\hline $\begin{array}{l}\text { Eye movement } \\
\text { defects }\end{array}$ & No & $\begin{array}{l}\text { Reduced lateral and } \\
\text { upward gaze }\end{array}$ & No & No & No & No & No \\
\hline Contractures & No & Heels & No & Heels & Heels & No & No \\
\hline CK level & $10 x$ & $6 x$ & $8 x$ & $4 x$ & n.a. & Normal & $15 x$ \\
\hline Histology & $\begin{array}{l}\text { TAs (mainly type II) fibre size } \\
\text { variability, internal nuclei, } \\
\text { fibrosis }\end{array}$ & $\begin{array}{l}\text { TAs, fibre size variability, } \\
\text { internal nuclei, fibrosis }\end{array}$ & $\begin{array}{l}\text { TAs mainly (type II) fibre } \\
\text { size variability, internal } \\
\text { nuclei }\end{array}$ & $\begin{array}{l}\text { TAs, fibre size variability, type I } \\
\text { fibre predominance, internal nuclei, } \\
\text { fibrosis }\end{array}$ & $\begin{array}{l}\text { TAs, fibre size variability, type I } \\
\text { fibre predominance, internal nuclei, } \\
\text { fibrosis }\end{array}$ & $\begin{array}{l}\text { TAs, type II } \\
\text { fibre atrophy }\end{array}$ & $\begin{array}{l}\text { TAs, type I fibre } \\
\text { predominance, type II fibre } \\
\text { atrophy }\end{array}$ \\
\hline $\begin{array}{l}\text { Tubular aggregate } \\
\text { classification }\end{array}$ & VMC & Ic & Ic & n.a. & $\mathrm{Ib}$ and $\mathrm{Ic}$ & n.a. & VMC and IC \\
\hline Additional features & $\begin{array}{l}\text { Postexercise myalgia and } \\
\text { fatigability, calf hypertrophy }\end{array}$ & Myalgia & $\begin{array}{l}\text { Myalgia, reduced tendon } \\
\text { reflexes }\end{array}$ & Myalgia & Myalgia & Myalgia & $\begin{array}{l}\text { Postexercise fatigability, calf } \\
\text { hypertrophy, episodic diplopia }\end{array}$ \\
\hline
\end{tabular}

TAs, tubular aggregates (Classification: Ib=tubular aggregates with central tubule; Ic=tubular aggregates with empty or moderately dense flocculent material); VMC=vesicular membrane collection n.a., not assessed; TAM, tubular aggregate myopathy. 
Figure 4 Histology of muscle biopsies from the patients with tubular aggregate myopathy. Histological analysis revealed tubular aggregates on modified Gomori trichrome and NADH tetrazolium reductase (NADH-TR) staining, but not on succinate dehydrogenase (SDH) staining. Internal nuclei are often seen.

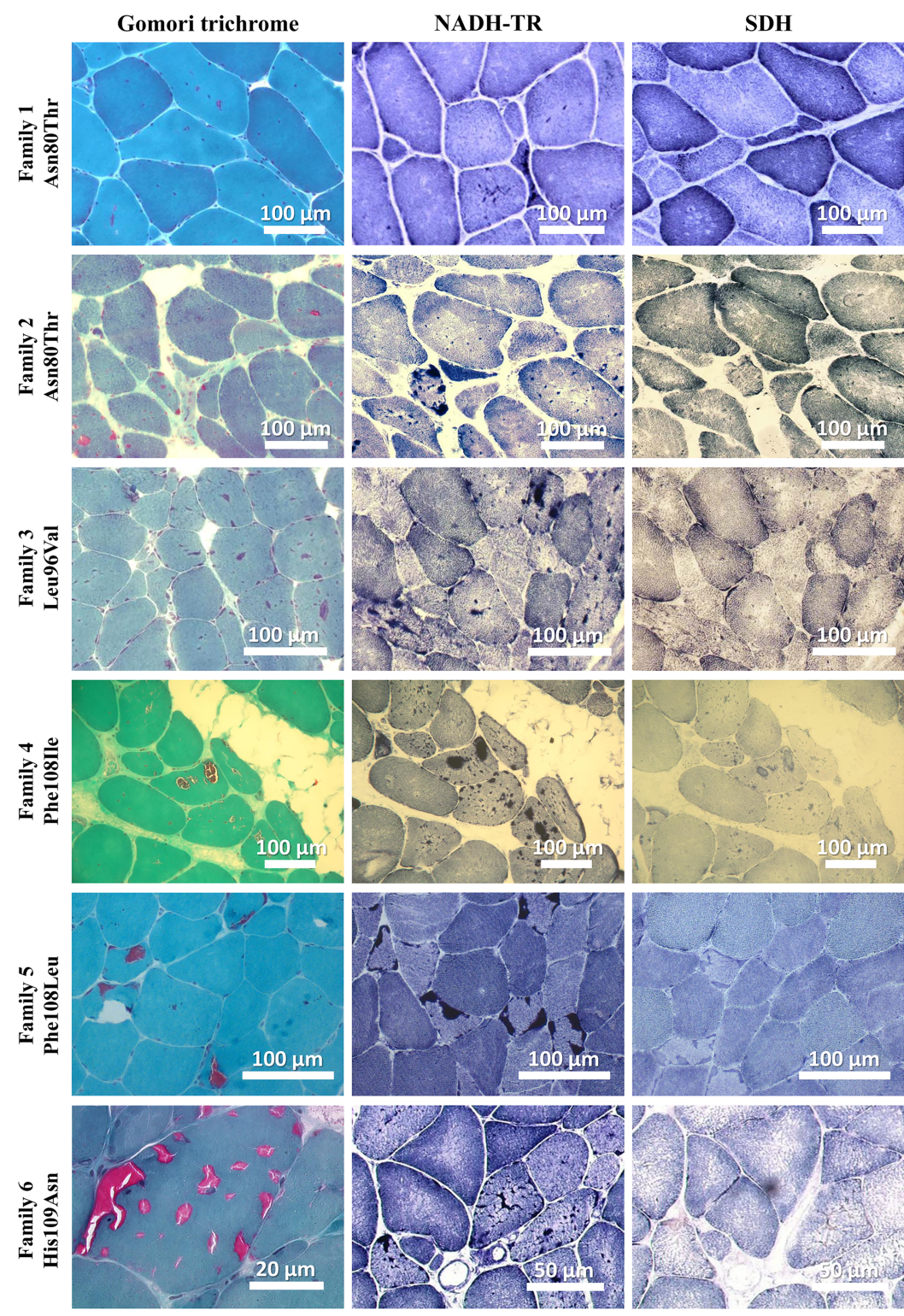

mutations potentially indicates mutation-dependent pathomechanism underlying the muscle disease.

Of note, the novel STIM1 p.Phe108Leu mutation was found to have a reduced effect at the molecular level, as constitutive STIM1 clustering was moderate in comparison with all other mutants. The patient is the only one with a normal CK level, had a disease onset in the $40 \mathrm{~s}$ and presents myalgia as the only clinical sign at age 52 years. This mild muscle phenotype might reflect the less pronounced impact of the mutation on STIM1 clustering.

\section{Mutation spectrum}

Including this study, STIM1 mutations have been found in 13 unrelated TAM families, supporting STIM1 as a major gene implicated in TAM. In total, nine different missense mutations affecting seven residues in the highly conserved STIM1 EF hands have been found in patients with TAM. The residues Phe108 and especially His109 appear to be prone to mutations and potentially represent a TAM hotspot, as they were mutated in seven families. The fact that all mutations reside in STIM1 exons 2 and 3 might direct and ease molecular diagnosis for patients with tubular aggregate myopathy and muscle weakness or myalgia. Importantly, our cohort encompassed 32 TAM families without mutations in STIM1, strongly suggesting the implication of yet unknown genes in the development of the muscle disorder. There is no obvious clinical and histological difference compared with the TAM families with STIM1 mutations.

Homozygous loss-of-function mutations in STIM1 were previously associated with immunodeficiency and neonatal hypotonia. ${ }^{11-13}$ Very recently, two groups reported in parallel the identification of the heterozygous p.Arg304Trp missense mutation in the cytosolic domain of STIM1 as the genetic cause of Stormorken syndrome, encompassing muscle weakness with tubular aggregates, miosis, thrombocytopenia, ichthyosis, mild intellectual disabilities, short stature and asplenia. ${ }^{14}{ }^{15}$ Further functional analysis and the characterisation of animal models will be needed to understand how different mutations in STIM1 cause such a phenotypical variability and a tissue-specific impact.

\section{Histological and ultrastructural characterisation}

Tubular aggregates were mainly reported in men from recessive or sporadic families and most often found only in type II fibres. $^{25}$ However, the present and previous studies on STIM1-related tubular aggregate myopathy involved a 
Figure 5 Electron microscopy of muscle biopsies from the patients with tubular aggregate myopathy. Ultrastructural analysis demonstrated prominent tubular aggregation with single-walled or double-walled membranes on transversal and longitudinal sections.
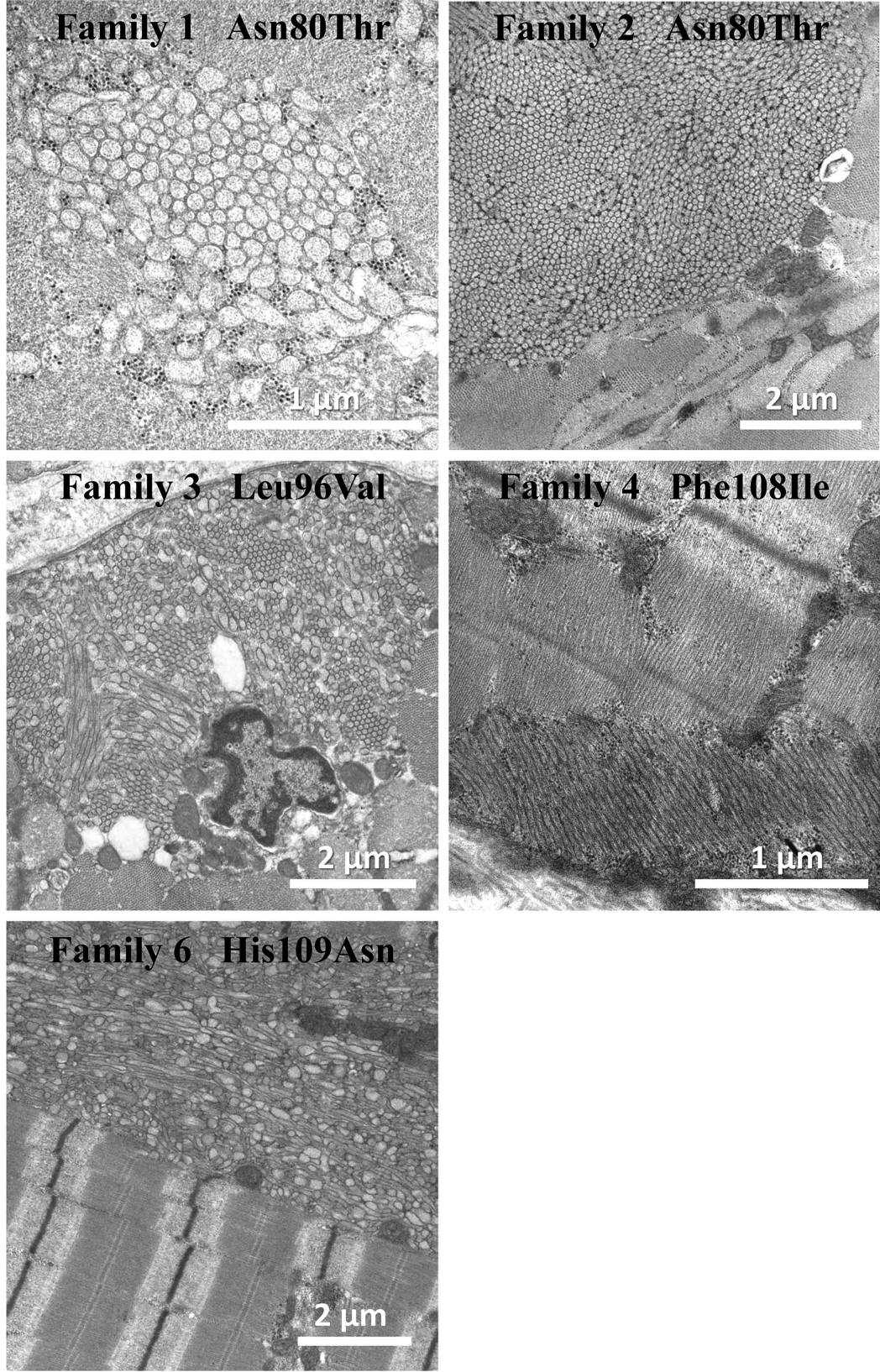

comparable number of male and female patients with equal distribution of aggregates in fibre types. It is possible that the TAM forms predominantly affecting type II fibres in men are caused by mutations in other genes.

\section{Pathological mechanisms}

All TAM-related STIM1 mutations identified to date affect highly conserved amino acids in the intraluminal $\mathrm{Ca}^{2+}$-sensing domain. According to previous studies, ${ }^{5} 9162627$ we demonstrated that all novel STIM1 mutations induce STIM1 clustering independently of drug-mediated $\mathrm{Ca}^{2+}$ store depletion. These data suggest that the mutants are unable to sense the calcium in the SR lumen, and constitutively cluster at the SR-plasma membrane junctions. The clusters are presumably functionally active, as they reside within normally structured sarcoplasmic reticulum without being positive for ubiquitin or p62. STIM1 clustering has been shown to constitutively activate the calcium entry channels and to strongly alter cellular $\mathrm{Ca}^{2+}$ homoeostasis. These findings strengthen the concept that the gain-of-function mutations disrupt the $\mathrm{Ca}^{2+}$-dependent activation/inactivation of STIM1, resulting in aberrant calcium homoeostasis, and ultimately in myalgia or muscle weakness.

Tubular aggregates originate from the SR, ${ }^{18} 2324$ and it has been proposed that sequential formation of aggregates in ageing skeletal muscle fibres is initiated by swelling of the SR cisternae and their extension into longitudinally oriented tubules. ${ }^{22}$ Different proteins involved in the uptake and the storage of $\mathrm{Ca}^{2+}$ such as STIM1, SERCA1, RYR1, sarcalumenin or triadin were previously shown to be components of the aggregates. ${ }^{16} 18$ The swelling of the SR in tubular aggregate myopathy potentially results from the excess of intraluminal and/or cytosolic $\mathrm{Ca}^{2+}$ as measured in myoblasts from a patient with TAM. ${ }^{16}$ Alternatively, tubular aggregate formation could be triggered by excessive STIM1 oligomerisation.

\section{CONCLUSION}

In conclusion, STIM1 mutations can cause a wide phenotypical spectrum ranging from childhood-onset muscle weakness to 
adult-onset myalgia. This is of major importance for genetic diagnosis and counselling as STIM1 mutations should be considered for patients with TAM presenting progressive muscle weakness, and for patients with myalgia as the first and predominant clinical sign. This study significantly broadens the phenotypical spectrum of muscle disorders caused by STIM1 mutations.

\section{Author affiliations}

${ }^{1}$ Department of Translational Medicine and Neurogenetics, IGBMC (Institut de Génétique et de Biologie Moléculaire et Cellulaire), Illkirch, France

${ }^{2}$ Inserm, U964, Illkirch, France

${ }^{3}$ CNRS, UMR7104, Illkirch, France

${ }^{4}$ University of Strasbourg, Illkirch, France

${ }^{5}$ Collège de France, Chaire de Génétique Humaine, Illkirch, France

${ }^{6}$ Department of Neuropathology, University Hospital Erlangen, Erlangen, Germany

${ }^{7}$ Neuromuscular Diseases and Neuroimmunology Unit, Fondazione IRCCS Istituto

Neurologico C. Besta, Milano, Italy

${ }^{8}$ Muscle Cell Biology Lab, Fondazione IRCCS Istituto Neurologico C. Besta, Milano, Italy

${ }^{9}$ Child Neurology Department, Fondazione IRCCS Istituto Neurologico C. Besta,

Milano, Italy

${ }^{10}$ Unit for Neuromuscular and Neurodegenerative Disorders, Laboratory of Molecular Medicine, Bambino Gesù Children's Hospital, Rome, Italy

${ }^{11}$ Institute of Neurology, Catholic University School of Medicine, Rome, Italy

${ }^{12}$ Neurology Department, Centre de Référence des Maladies Neuromusculaires,

Centre Hospitalier Universitaire d'Angers, Angers, France

${ }^{13}$ Centre de Référence de Pathologie Neuromusculaire Paris-Est, Groupe Hospitalier Pitié-Salpêtrière, Paris, France

${ }^{14}$ Institut de Myologie, GHU La Pitié-Salpêtrière, Paris, France

${ }^{15}$ Department of Neurology, Martin-Luther-University Halle-Wittenberg, Halle (Saale), Germany

Acknowledgements The authors thank the members of the families for their cooperation and interest in this study, EuroBioBank and the Telethon network of genetic biobanks (grant n. GTB12001F to M.M.) Nicolas Demaurex, Marc Koch, Catherine Fressinaud and Stéphanie Bauché-Godard for material supply, technical assistance and discussions. None of the authors reports conflicts of interest.

Contributors $J B$ and $J L$ designed and coordinated the study. JB, FC, CK, GAP and FF performed the experiments. MM, LM, BP, IM, GT, ER, IP-B, AN-P, MF, PRJ, MD, NBR and BE contributed clinical samples, histological and patient data. JB and JL wrote the manuscript.

Funding This work was supported by grants from Institut National de la Santé et de la Recherche Médicale (INSERM), Centre National de la Recherche Scientifique (CNRS), University of Strasbourg, Collège de France, Agence Nationale de la Recherche (ANR-11-BSV1-026-01 and ANR-10-LABX-0030-INRT), Muscular Dystrophy Association, and Association Francaise contre les Myopathies (AFM).

Competing interests None.

Patient consent Obtained.

Ethics approval Comité de Protection des Personnes (DC-2012-1693).

Provenance and peer review Not commissioned; externally peer reviewed.

\section{REFERENCES}

1 Li Z, Lu J, Xu P, Xie X, Chen L, Xu T. Mapping the interacting domains of STIM1 and Orai1 in $\mathrm{Ca} 2+$ release-activated $\mathrm{Ca} 2+$ channel activation. J Biol Chem 2007:282:29448-56.

2 Muik M, Fahrner M, Schindl R, Stathopulos P, Frischauf I, Derler I, Plenk P, Lackner B, Groschner K, Ikura M, Romanin C. STIM1 couples to ORAl1 via an intramolecular transition into an extended conformation. EMBO J 2011;30:1678-89.

3 Stathopulos PB, Zheng L, Li GY, Plevin MJ, Ikura M. Structural and mechanistic insights into STIM1-mediated initiation of store-operated calcium entry. Cell 2008:135:110-22.

4 Stathopulos PB, Li GY, Plevin MJ, Ames JB, Ikura M. Stored Ca2+ depletion-induced oligomerization of stromal interaction molecule 1 (STIM1) via the EF-SAM region: an initiation mechanism for capacitive $\mathrm{Ca} 2+$ entry. $J$ Biol Chem 2006:281:35855-62

5 Zhang SL, Yu Y, Roos J, Kozak JA, Deerinck TJ, Ellisman MH, Stauderman KA, Cahalan MD. STIM1 is a Ca2+ sensor that activates CRAC channels and migrates from the $\mathrm{Ca} 2+$ store to the plasma membrane. Nature 2005;437:902-5.

6 Xu P, Lu J, Li Z, Yu X, Chen L, Xu T. Aggregation of STIM1 underneath the plasma membrane induces clustering of Orai1. Biochem Biophys Res Commun 2006;350:969-76.
7 Wu MM, Buchanan J, Luik RM, Lewis RS. Ca2+ store depletion causes STIM1 to accumulate in ER regions closely associated with the plasma membrane. J Cell Biol 2006; 174:803-13.

8 Luik RM, Wu MM, Buchanan J, Lewis RS. The elementary unit of store-operated Ca2+ entry: local activation of CRAC channels by STIM1 at ER-plasma membrane junctions. J Cell Biol 2006;174:815-25.

9 Liou J, Kim ML, Heo WD, Jones JT, Myers JW, Ferrell JE Jr, Meyer T. STIM is a Ca2+ sensor essential for Ca2+-store-depletion-triggered Ca2+ influx. Curr Biol 2005; 15:1235-41.

10 Liou J, Fivaz M, Inoue T, Meyer T. Live-cell imaging reveals sequential oligomerization and local plasma membrane targeting of stromal interaction molecule 1 after Ca2+ store depletion. Proc Natl Acad Sci USA 2007;104:9301-6.

11 Byun M, Abhyankar A, Lelarge V, Plancoulaine S, Palanduz A, Telhan L, Boisson B, Picard C, Dewell S, Zhao C, Jouanguy E, Feske S, Abel L, Casanova JL. Whole-exome sequencing-based discovery of STIM1 deficiency in a child with fatal classic Kaposi sarcoma. J Exp Med 2010;207:2307-12.

12 Fuchs S, Rensing-Ehl A, Speckmann C, Bengsch B, Schmitt-Graeff A, Bondzio I, Maul-Pavicic A, Bass T, Vraetz T, Strahm B, Ankermann T, Benson M, Caliebe A, Fölster-Holst R, Kaiser P, Thimme R, Schamel WW, Schwarz K, Feske S, Ehl S. Antiviral and regulatory $T$ cell immunity in a patient with stromal interaction molecule 1 deficiency. J Immunol 2012;188:1523-33.

13 Picard C, McCarl CA, Papolos A, Khalil S, Luthy K, Hivroz C, LeDeist F, Rieux-Laucat F, Rechavi G, Rao A, Fischer A, Feske S. STIM1 mutation associated with a syndrome of immunodeficiency and autoimmunity. $N$ Engl I Med 2009;360:1971-80.

14 Misceo D, Holmgren A, Louch WE, Holme PA, Mizobuchi M, Morales RJ, De Paula AM, Stray-Pedersen A, Lyle R, Dalhus B, Christensen G, Stormorken H, Tjønnfjord $\mathrm{GE}$, Frengen $\mathrm{E}$. A dominant STIM1 mutation causes Stormorken syndrome. Hum Mutat 2014.

15 Nesin V, Wiley G, Kousi M, Ong EC, Lehmann T, Nicholl DJ, Suri M, Shahrizaila N, Katsanis N, Gaffney PM, Wierenga KJ, Tsiokas L. Activating mutations in STIM1 and ORAl1 cause overlapping syndromes of tubular myopathy and congenital miosis. Proc Natl Acad Sci USA 2014

16 Bohm J, Chevessier F, Maues De Paula A, Koch C, Attarian S, Feger C, Hantai D, Laforet $\mathrm{P}$, Ghorab K, Vallat JM, Fardeau M, Figarella-Branger D, Pouget J, Romero NB, Koch M, Ebel C, Levy N, Krahn M, Eymard B, Bartoli M, Laporte J. Constitutive activation of the calcium sensor STIM1 causes tubular-aggregate myopathy. Am J Hum Genet 2013;92:271-8.

17 Hedberg C, Niceta M, Fattori F, Lindvall B, Ciolfi A, D'Amico A, Tasca G, Petrini S, Tulinius M, Tartaglia M, Oldfors A, Bertini E. Childhood onset tubular aggregate myopathy associated with de novo STIM1 mutations. J Neurol 2014.

18 Chevessier F, Bauche-Godard S, Leroy JP, Koenig J, Paturneau-Jouas M, Eymard B, Hantai D, Verdiere-Sahuque M. The origin of tubular aggregates in human myopathies. J Pathol 2005;207:313-23.

19 Sternberg D, Maisonobe T, Jurkat-Rott K, Nicole S, Launay E, Chauveau D, Tabti N, Lehmann-Horn F, Hainque B, Fontaine B. Hypokalaemic periodic paralysis type 2 caused by mutations at codon 672 in the muscle sodium channel gene SCN4A. Brain 2001:124(Pt 6):1091-9.

20 Belaya K, Finlayson S, Slater CR, Cossins J, Liu WW, Maxwell S, McGowan SJ, Maslau S, Twigg SR, Walls TJ, Pascual Pascual SI, Palace J, Beeson D. Mutations in DPAGT1 cause a limb-girdle congenital myasthenic syndrome with tubular aggregates. Am J Hum Genet 2012;91:193-201.

21 Guergueltcheva V, Muller JS, Dusl M, Senderek J, Oldfors A, Lindbergh C, Maxwell S, Colomer J, Mallebrera CJ, Nascimento A, Vilchez JJ, Muelas N, Kirschner J, Nafissi S, Kariminejad A, Nilipour Y, Bozorgmehr B, Najmabadi H, Rodolico C, Sieb JP, Schlotter B, Schoser B, Herrmann R, Voit T, Steinlein OK, Najafi A, Urtizberea A Soler DM, Muntoni F, Hanna MG, Chaouch A, Straub V, Bushby K, Palace J, Beeson D, Abicht A, Lochmüller H. Congenital myasthenic syndrome with tubular aggregates caused by GFPT1 mutations. J Neurol 2011.

22 Boncompagni S, Protasi F, Franzini-Armstrong C. Sequential stages in the age-dependent gradual formation and accumulation of tubular aggregates in fast twitch muscle fibers: SERCA and calsequestrin involvement. Age (Dordr) 2012:34:27-41.

23 Engel WK, Bishop DW, Cunningham GG. Tubular aggregates in type II muscle fibers: ultrastructural and histochemical correlation. J Ultrastruct Res 1970;31:507-25.

24 Muller HD, Vielhaber S, Brunn A, Schroder JM. Dominantly inherited myopathy with novel tubular aggregates containing 1-21 tubulofilamentous structures. Acta Neuropathol 2001;102:27-35.

25 Schiaffino $S$. Tubular aggregates in skeletal muscle: Just a special type of protein aggregates? Neuromuscul Disord 2012;22:199-207.

26 Mercer JC, Dehaven WI, Smyth JT, Wedel B, Boyles RR, Bird GS, Putney JW Jr. Large store-operated calcium selective currents due to co-expression of Orai1 or Orai2 with the intracellular calcium sensor, Stim1. J Biol Chem 2006;281:24979-90.

27 Huang GN, Zeng W, Kim JY, Yuan JP, Han L, Muallem S, Worley PF. STIM1 carboxyl-terminus activates native SOC, I(crac) and TRPC1 channels. Nat Cell Biol 2006;8:1003-10. 


\section{JMG}

Clinical, histological and genetic characterisation of patients with tubular aggregate myopathy caused by mutations in STIM1

Johann Böhm, Frédéric Chevessier, Catherine Koch, G Arielle Peche, Marina Mora, Lucia Morandi, Barbara Pasanisi, Isabella Moroni, Giorgio Tasca, Fabiana Fattori, Enzo Ricci, Isabelle Pénisson-Besnier, Aleksandra Nadaj-Pakleza, Michel Fardeau, Pushpa Raj Joshi, Marcus Deschauer, Norma Beatriz Romero, Bruno Eymard and Jocelyn Laporte

J Med Genet 2014 51: 824-833 originally published online October 17, 2014

doi: 10.1136/jmedgenet-2014-102623

Updated information and services can be found at:

http://jmg.bmj.com/content/51/12/824

These include:

References This article cites 23 articles, 10 of which you can access for free at: http://jmg.bmj.com/content/51/12/824\#BIBL

Email alerting Receive free email alerts when new articles cite this article. Sign up in the service box at the top right corner of the online article.

Topic Articles on similar topics can be found in the following collections Collections

\section{Notes}

To request permissions go to:

http://group.bmj.com/group/rights-licensing/permissions

To order reprints go to:

http://journals.bmj.com/cgi/reprintform

To subscribe to BMJ go to:

http://group.bmj.com/subscribe/ 